Immunogenetics 1:184-188, 1974

(C) by Springer-Verlag New York Inc. 1974

Brief Communication

\title{
Genetic Nomenclature for the $H-2$ Complex of the Mouse
}

\author{
J. Klein 1, F. H. Bach ${ }^{2}$, F. Festenstein ${ }^{3}$, H. O. McDevitt ${ }^{4}$, D. C. Shreffler ${ }^{5}$, \\ G. D. Snell6, and J. H. Stimpfling7
}

1 The University of Texas Southwestern Medical School, Dallas, Texas

2 The University of Wisconisn, Madison, Wisconsin

${ }^{3}$ The London Hospital Medical College, London, England

${ }^{4}$ Stanford University School of Medicine, Stanford California

5 The University of Michigan Medical School, Ann Arbor, Michigan

6 The Jackson Laboratory, Bar Harbor, Maine

7 McLaughlin Research Institute, Great Falls, Montana

Received December 3, 1973

\section{Regions of the $H-2$ Complex}

The $H-2$ complex (system, Snell and Stimpfling 1966) represents a segment of chromosome 17 delimited by the $H-2 K$ and $H-2 D$ loci (Table 1). The complex is split by the $S s$ locus into two ends, the $\mathbf{K}$ end between $H-2 K$ and $S s$, and the $\mathbf{D}$ end between $S s$ and $H-2 D$. The term end can also be used to indicate the direction; thus the $\mathrm{K}$ end could mean the direction from the $S s$ locus toward the centromere, and the $D$ end the direction from the $S s$ locus toward the telomere. The $H-2$ complex is composed of regions, subregions and loci. An $H$-2 region is a segment of the $H-2$ complex delimited by recombination and consisting of a marker gene and an undeterminate number of neighboring loci. The $H-2$ complex is to be divided into four regions, $K, I, S$ and $D$, defined by marker genes $H$-2K, $I r-1 A, S$ s and $H$-2D, respectively. Subregions of a given region are segments identified by distinct but functionally related marker genes and separated from each other and the other regions by recombination. The $I$ region is to be divided into two subregions, $I r-1 A$ and $I r-I B$, formerly termed $I r-1$ and $I r-I g G$, respectively. It is assumed that each region or subregion contains at least one locus,

This document is the outcome of the discussions held during the H-2 Workshop in Bar Harbor, Maine on October $8-10,1973$. These discussions were instigated by the organizers of the Workshop, Drs. Ian F. C. McKenzie and Marianna Cherry, to whom we wish to express our gratitude for their encouragments and support. H. F. wishes to acknowledge travel support from the Wellcome Trust Foundation. 
Table 1. Genetic Map of the H-2 Complex

\begin{tabular}{|c|c|c|c|c|c|}
\hline Complex & \multicolumn{5}{|c|}{$\mathrm{H}-2$} \\
\hline Ends & \multicolumn{3}{|c|}{$K$} & \multicolumn{2}{|c|}{$D$} \\
\hline Regions & $K$ & \multicolumn{2}{|c|}{$I$} & $S$ & $D$ \\
\hline Subregions & & $\operatorname{Ir}-1 A$ & $\operatorname{Ir}-1 B$ & & \\
\hline Loci & $H-2 K$ & $I r-1 A$ & $I r-1 B$ & $S s, S l p$ & $H-2 D$ \\
\hline & & $H-2 I$ & & & \\
\hline
\end{tabular}

defined as a segment of the genetic material coding for a single polypeptide chain. The postulated loci in the known $H-2$ regions and subregions are designated $H-2 K, I r-1 A$, $I r-1 B, S s, S l p$, and $H-2 D$. The terms region and subregion are necessary because the number of loci in a chromosomal segment separable by recombination remains unknown.

The alternate forms of genes at a single locus are called alleles and are designated by small superscript letters which indicate their genetic origin (e.g., $H-2 K \mathrm{k}, H-2 D$ k, $S s^{\text {b }}$, etc.). No suitable name is available for the alternate forms of individual regions and subregions; if necessary, they too can be called alleles and designated by superscript letters. In tables and charts where there is no risk of confusion, the subregion symbols can be omitted and the alternate forms designated by small letters in the same order as they appear on the genetic map (e.g., the alleles of the $H-2 K, I r-I A$, $I r-1 B, S s$ and $H-2 D$ loci in the $H-2$ a complex are $k k k d d)$. The alternative forms of the whole $H-2$ complex are to be called haplotypes (replacing the former terms alleles and chromosomes).

\section{Haplotype Symbolism}

The $H-2$ haplotypes are to be designated $H-2$, with a superscript consisting of one or two small letters and, in some instances, an Arabic numeral (e.g., $H-2 b, H-2 d a$, $H-2^{h l}, H-2 a p I$, etc.). Since all single letters of the alphabet have been exhausted (that is, all except $H-2^{x}$ reserved for designation of unknown $H-2$ haplotypes), it has been necessary to resort to double letters in naming new haplotypes. Any combination of double letters is permissible as long as it is cleared through the $H$ - 2 registry (currently maintained by Dr. Peter Démant, Institute of Experimental Biology and Genetics, Budejovická 1083, PrahaKrc $\stackrel{\vee}{r}$, Czechoslovakia) to assure that it has not been used before. For the second letter of the superscript, the first half of the alphabet is reserved for minor variants (e.g., $\mathrm{H}-2^{b a}$ is a minor variant of $H-2^{b}$ ), whereas the second half of the alphabet should be used for new major $H-2$ haplotypes (Snell et al 1964). The haplotype symbols of the $H-2$ recombinants with identical $H-2 K$ and $H-2 D$ regions should consist of the same single or double letters followed by an Arabic numeral to distinguish the individual recombinants. The letter "l" and the numeral " 0 " should not be used to avoid confusion with numeral " 1 " and letter "o", respectively. The first recombinant in each series should be designated with numeral " 1 " 
(the three exceptions from this rule are $H$-2 haplotypes $g, h$, and $i$ which remain without numerals). The haplotypes of wild mice are to be designated by the superscript letter " $w$ ", followed by an Arabic numeral assigned in the order of discovery (e.g., $H-2 w l, H-2 w 2, H-2 w 3$, etc.). In accordance with the new rules, some of the currently existing haplotype symbols are changed as indicated in Table 2. For description of the haplotype's origin, and for references, see Klein 1974.

Table 2. Recommended Changes in the Designation of Some $H$-2 Haplotypes

\begin{tabular}{|c|c|c|}
\hline $\begin{array}{l}\text { Recommended } \\
\text { Symbol }\end{array}$ & Old Symbol & Type Strain \\
\hline$a l$ & al & A.AL \\
\hline an 1 & te & A.TFR 1 \\
\hline ap1 & $a p$ & $\mathrm{~B} 10 . \mathrm{M}(11 \mathrm{R})$ \\
\hline$a p 2$ & $t a$ & A.TFR2 \\
\hline ap3 & $t b$ & A.TFR3 \\
\hline ap 4 & $t c$ & A.TFR4 \\
\hline ap5 & $t d$ & A.TFR5 \\
\hline$a q 1$ & $a q$ & $\mathrm{~B} 10 . \mathrm{M}(17 \mathrm{R})$ \\
\hline$g$ & $g-G o$ & HTG \\
\hline g1 & $g-E g$ & B10.D2(R101) \\
\hline$g 2$ & $g d$ & $\mathrm{D} 2 . \mathrm{GD}$ \\
\hline$g 3$ & $g-2 E g$ & $\mathrm{~B} 10 . \mathrm{D} 2(\mathrm{R} 103)$ \\
\hline$g 4$ & $g e$ & B $10 . B D R 1$ \\
\hline$g 5$ & $g f$ & B10.BDR2 \\
\hline$h$ & $h-G o$ & HTH \\
\hline$h l$ & $h-S g$ & $\mathrm{~B} 10 . \mathrm{A}(1 \mathrm{R})$ \\
\hline$h 2$ & $h-2 S g$ & $B 10 . A(2 R)$ \\
\hline$h 3$ & $h g$ & B10.AM \\
\hline$h 4$ & $h-3 S g$ & B10.A(4R) \\
\hline$h 15$ & $h-4 S g$ & $\mathrm{~B} 10 . \mathrm{A}(15 \mathrm{R})$ \\
\hline$h 18$ & $h-5 S g$ & B10.A (18R) \\
\hline$i$ & $i-G o$ & HTI \\
\hline$i 3$ & $i-S g$ & $\mathrm{~B} 10 . \mathrm{A}(3 \mathrm{R})$ \\
\hline$i 5$ & $i-2 S g$ & $\mathrm{~B} 10 . \mathrm{A}(5 \mathrm{R})$ \\
\hline$i 7$ & $i-E g$ & $\mathrm{~B} 10 . \mathrm{D} 2(\mathrm{R} 107)$ \\
\hline$i a 1$ & $i a$ & $\mathrm{~B} 10 . \mathrm{D} 2(\mathrm{R} 106)$ \\
\hline$o l$ & ol & C3H.OL \\
\hline$o 2$ & oh & C3H.OH \\
\hline$q p 1$ & $q s$ & $\mathrm{DA}$ \\
\hline$s q 1$ & $a r$ & $\begin{array}{l}\text { A. QSR } 1 \\
\quad \text { (Continued) }\end{array}$ \\
\hline
\end{tabular}


Table 2. (Continued)

\begin{tabular}{ccl}
\hline $\begin{array}{c}\text { Recommended } \\
\text { Symbol }\end{array}$ & Old Symbol & \multicolumn{1}{c}{ Type Strain } \\
\hline$s q 2$ & $a s$ & B10.QSR2 \\
$t 1$ & $t l$ & A.TL \\
$t 2$ & $t h$ & B 10.S(7R) \\
$t 3$ & $t t$ & B 10.HTT \\
$w 1$ & $w a$ & B 10.KPA42 \\
$w 2$ & $w b$ & B 10.KPB68 \\
$w 3$ & $w c$ & B 10.SAA48 \\
$w 4$ & $w d$ & B 10.GAA20 \\
$w 5$ & $w e$ & B10.KEA5 \\
$w 6$ & $w f$ & B 10.TOB1 \\
$y 1$ & $y-K l j$ & B10.AQR \\
$y 2$ & $y-S g$ & B10.T(6R). \\
\hline
\end{tabular}

\section{Determinants for Various H-2 Associated Functions}

In a number of instances, the same region or subregion has been associated with several different functions. For example, the $I r-1 A$ subregion not only determines the immune response to synthetic polypeptides, (T, G)-A-L, (Phe, G)-A--L and (H, G)A--L, but it also controls lymphocyte activation in mixed lymphocy te culture (MLC), graft-versus-host reaction (GVHR), skin graft rejection, etc. These functions may be governed by distinct loci not yet separated by recombination or they may represent the pleiotropic effects of a single gene. Since it may take some time before one of these two alternatives is experimentally proved, it is felt to be desirable that distinct loci be postulated for different functions. These loci could, therefore, be assigned distinct genetic symbols. Should it later prove that some of the loci are actually identical, only one locus symbol will be retained (on the basis of priority), and the other symbols will be dropped. The suggested genetic symbols for the different functions are as follows.

Serologically and Histogenetically Defined Loci. Genetic loci responsible for production of humoral $H-2$ antibodies and/or (skin) graft rejection should be designated by the prefix $H-2$ followed by a single capital letter. It is presumed that the serologically and histogenetically detectable loci are identical, although this presumption has not yet been proved. The $H$ symbol is assigned for historical reasons. At present, three histocompatibility loci are known to exist in the $H-2$ complex. $H-2 K$, $H-2 I$ and $H-2 D$, located in the $K, I$ and $D$ regions, respectively.

Immune Response (Ir) Loci. Whenever they are shown to be separable by recombination, the $H$-2-associated $I r$ loci should be given symbols consisting of $I r-1$ followed by a capital letter (i.e., $\operatorname{Ir}-1 A, \operatorname{Ir}-1 B, \operatorname{Ir}-1 C, \ldots \operatorname{Ir}-1 Z, \operatorname{Ir}-1 A A, \operatorname{Ir}-1 A B, \ldots$ $I r-1 A Z$, etc.). The letters should be assigned in the order of discovery of the $I r-1$ loci. In accordance with this rule it is suggested that the symbols of the two presently known $I r$ loci be changed from $I r-1$ to $I r-1 A$, and from $I r-I g G$ to $I r-1 B$. The $H-2$ - 
associated immune response determinants not yet separated by recombination (i.e., those mapping in either the $I r-1 A$ or $I r-1 B$ subregions) should not be assigned standard gene symbols but instead be referred to by provisional symbols consisting of the prefix $I r$ and an abbreviation of the antigen to which a particular immune response is directed (e.g., $I r$ - $O A$ stands for immune responsiveness to ovalbumin, $I r-B G G$ for responsiveness to bovine gamma globulin, etc.). If the immune response to a given antigen later proves to be controlled by one of the already known Ir-1 loci, the provisional symbol will be dropped; if, on the other hand, the responsiveness proves to be controlled by a separate locus, the provisional symbol will be replaced by a genetic symbol in the $I r-1$ series (i.e., $I r-1 C, I r-1 D$, etc.). The alternative forms (alleles) at the $I r-I$ loci or subregions are designated by small superscript letters indicating their genetic origin (e.g. $I r-1 A^{b}$ and $I r-1 B^{b}$ in the case of the $H-2^{b}$ haplotype; $I r-1 A^{k}$ and $I r-1 B^{k}$ in the case of the $H-2^{a}$ haplotype, etc.).

Loci for I-Region-Associated Antigens. Loci residing in the $I$ region and controlling antigens preferentially expressed on lymphocytes are designated by the $I a$ symbol, as described in the accompanying article. (Shreffler et al. 1974).

Mixed lymphocyte culture (MLC) and graft-versus-host (GVH) reactivity. It has been established that there are loci in the $H-2$ complex which are of prime importance in controlling both MLC and GVH reactivity. These loci are separable from the $H-2 K$ and $H-2 D$ loci. Details of nomenclature for these loci are currently under discussion by the investigators concerned and will be dealt with in a later article.

\section{References}

Klein, J.: Biology of the Mouse Histocompatibility-2 Complex. Springer-Verlag, New York, N. Y., in press 1974.

Shreffler, D., David, C., Götze, D., Klein, J., McDevitt, H., and Sachs, D.: Genetic nomenclature for new lymphocyte antigens controlled by the $I$ region of the H-2 complex. Immunogenetics 1:189-190 1974.

Snell, G. D. and Stimpfling, J. H.: Genetics of tissue transplantation. In: E. L. Green (ed.): Biology of the Laboratory Mouse, pp. 457-491, McGraw-Hill, New York, N. Y., 1966.

Snell, G. D., Hoecker, G., Amos, D. B., and Stimpfling, J. H.: A revised nomenclature for the histocompatibility-2 locus of the mouse. Transplantation 2:777-784, 1967. 